\title{
Possível relação das alterações dietéticas de micronutrientes com a sintomatologia comportamental no distúrbio do espectro autista
}

\section{Possible relationship of dietary changes in micronutrients with behavioral symptoms in autistic spectrum disorder}

Nathália de Freitas Penaforte1, Carlos Augusto Carvalho de Vasconcelos ${ }^{2}$, Angélica de Kassia Barbosa Flôr ${ }^{3}$

${ }^{1}$ Discente da Faculdade de Comunicação, Tecnologia e Turismo de Olinda, Olinda, PE, Brasil.

${ }^{2}$ Docente do Departamento de Nutrição do Centro de Ciências da Saúde, Universidade Federal de Pernambuco, Recife, PE, Brasil.

${ }^{3}$ Docente do Departamento de Nutrição da Faculdade de Comunicação, Tecnologia e Turismo de Olinda, Olinda, PE, Brasil.

\section{Resumo}

Este trabalho teve como objetivo analisar a possível relação das alterações dietéticas de micronutrientes com a sintomatologia comportamental característica do Distúrbio do Espectro Autista (DEA), verificando que complicações podem estar envolvidas e o que isso pode impactar nesse público. Foi realizada uma revisão bibliográfica nas bases de dados eletrônicas "Pubmed", "Science Direct" e "Scielo", publicados em inglês e português no período de janeiro de 2002 a outubro de 2019. Utilizou os seguintes descritores de busca: "autistic desorder" AND "vitamins" AND "minerals" AND "gastrointestinal tract" AND "nutrition" AND "behavior" e suas combinações. Na busca, foram encontrados 2.857 artigos, dentre os quais, mediante os critérios de inclusão e de exclusão apenas 47 artigos foram selecionados, e mais 2 livros. Observou-se que esse público tem uma maior probabilidade de ter uma inadequação de micronutrientes, em que pode ser heterogêneo, variando de deficiência a toxicidade, ocasionada pela dieta restritiva e monótona, refletindo assim no seu estado nutricional. A frequência de sintomas intestinais também pode estar atrelada ao agravamento do comportamento alimentar e da sintomatologia típica do transtorno, que consequentemente reflete de forma negativa na absorção desses nutrientes, os quais desempenham papéis cruciais no neurodesenvolvimento. Concluímos que é necessário a realização de novas pesquisas nesta área para avaliar a ingestão alimentar de micronutrientes específicos, para verificar se há comprometimento da absorção desses nutrientes, seu impacto no comportamento característico e na eficácia da suplementação validada neste público. Assim, possibilitando a criação de medidas preventivas, embora as causas do DEA ainda sejam incertas, a influência dos micronutrientes não deve ser negligenciada.

Palavras-chave: Autismo, Minerais, Vitaminas, Comportamento.

\begin{abstract}
Objective: Analyze in literature possible relationship of micronutrients dietary changes with behavioral symptoms characteristic of Autistic Spectrum Disorder (ASD), checking which complications may be affected and what may impact this public. Methods: A literature review was performed on the electronic databases "Pubmed", "Science Direct" and "Scielo", published from January 2002 to October 2019, in english and portuguese language. It used the following search descriptors: "autistic desorder" AND "vitamins" AND "minerals" AND "gastrointestinal tract" AND "nutrition" AND "behavior" and their combinations. In the search, 2857 articles were found, among which using the inclusion and exclusion criteria only 47 was included articles, as well as 2 more reference books. Results and Discussion: It is observed this public is more likely to have micronutrient inadequacy which may be heterogeneous, a variable difficulty in toxicity caused by a restrictive and monotonous diet, thus reflecting on their nutritional status. The frequency of intestinal symptoms may also be linked to the worsening eating behavior and the typical symptomatology of the disorder, which consequently is negatively reduced in the absorption of these
\end{abstract}


nutrients, acting crucial roles in neurological involvement. Conclusion: Further research is needed in this area to assess the dietary intake of specific micronutrients, verify if there is impairment of absorption of these nutrients, their impact on the characteristic behavior and the effectiveness of supplementation validated in this public. Thus, enabling the creation of preventive measures, although the causes of ASD are still uncertain the influence of micronutrients should not be overlooked.

Keywords: Autism. Minerals. Vitamins. Behavior.

\section{Introdução}

O distúrbio do espectro autista (DEA) se identifica pela presença da deficiência qualitativa da interação social recíproca, habilidades de comunicação deficiente e por interesses e comportamentos restritos, repetitivos e estereotípicos, sendo cada vez mais prevalentes síndromes comportamentais neurodesenvolvidas do comprometimento verbal e comunicação não verbal $^{1}$. Segundo pesquisadores, o cérebro de uma pessoa com DEA pode apresentar falhas de comunicação entre os neurônios, dificultando o processamento de informações, principalmente no corpo caloso, a amígdala e o cerebelo ${ }^{2}$.

Além disso, o desconforto gastrointestinal, inflamação intestinal, diarreia, constipação e refluxo ácido, são algumas alterações características nessas crianças que afeta seu estado nutricional, refletindo na capacidade de ingestão e absorção dos alimentos e nutrientes, respectivamente ${ }^{3}$. Enfatizando que crianças com DEA têm sido consideradas como um grupo de risco no desenvolver de possíveis carências nutricionais, principalmente de micronutrientes. Sendo a suplementação de vitaminas e minerais uma alternativa terapêutica por alterar a expressão de sintomas no DEA ${ }^{4}$.

Diante disso, esse estudo objetivou analisar na literatura disponível a possível relação das alterações dietéticas de micronutrientes com a sintomatologia comportamental característica do DEA, verificando que complicações podem estar envolvidas e o que isso pode impactar nesse público.

\section{Métodos}

Seleção das bases de pesquisa e estratégias de busca

O presente estudo foi realizado através de uma revisão bibliográfica de caráter exploratório utilizando-se artigos científicos, projetos de monografia, artigos publicados em revistas e livros conceituados de referência.Buscamos entender a possível relação das alterações dietéticas de micronutrientes com a sintomatologia comportamental no DEA. A pesquisa foi por meio das bases de dados eletrônicas "Pubmed", "Science Direct" e "Scielo" utilizando como ferramenta de estratégia os "Descritores em Ciências da Saúde (DeCS)" para selecionar os seguintes descritores de busca: "autistic desorder" AND "vitamins" AND "minerals" AND "gastrointestinal tract" AND "nutrition" AND "behavior" e suas combinações.

\section{Critérios de inclusão e de exclusão}

Como critérios de inclusão foram considerados estudos escritos em inglês e português publicados no período de janeiro de 2002 a outubro de 2019, com público em análise com diagnóstico confirmado do DEA e discussões a respeito do objetivo do artigo. Excluímos aqueles que tinham assuntos discordantes do objetivo e que não tivesse como foco a nutrição e/ou avaliação do consumo alimentar e comportamental. Cartas ao editor e relatos de casos foram excluídos da pesquisa.

\section{Análise dos dados}

A triagem inicial dos artigos consistiu na avaliação dos títulos e dos resumos, seguida de uma leitura minuciosa e crítica dos artigos selecionados para determinar quais preenchiam os critérios de inclusão e de exclusão.

$\mathrm{Na}$ busca, foram encontrados 2.857 artigos, sendo 609 no "Pubmed", 2.113 no "Science Direct" e 135 no "Scielo". Mediante os critérios de inclusão e de exclusão 47 artigos e 2 livros de referência foram incluídos na presente revisão.

\section{Resultados e Discussão}

Hábitos comportamentais 
Esses indivíduos podem apresentar inúmeros sintomas comportamentais, incluindo hiperatividade, desatenção, impulsividade, agressividade, comportamentos auto agressivos e, particularmente, em crianças mais jovens, acessos de raiva. Respostas incomuns a estímulos sensoriais, anormalidade na alimentação, humor, afeto ou sono podem ser observadas. Pode haver ausência de medo em respostas a perigos reais e temor excessivo em resposta a objetos inofensivos ${ }^{5}$.

Dentre outras comorbidades comumente associadas incluem alterações na função da barreira intestinal, autoimunidade e retardo mental, além de interferir no consumo alimentar ${ }^{6}$. Dentre esses sintomas podem-se classificar três categorias de comportamentos que vão afetar o perfil alimentar do autista, o comportamento alimentar seletivo (CAS), a recusa de alimentos, pelo fato de haver uma resistência a prova de novos alimentos e a indisciplina durante as refeições, fator que é bem característico na infância e no próprio transtorno. São esses fatores que fazem com que a criança com DEA possua uma dieta monótona e de variedade limitada, acarretando prováveis carências nutricionais ${ }^{7}$.

\section{Perfil alimentar}

A alimentação inadequada associada a falta de equilíbrio energético torna-se de fato uma preocupação em crianças com DEA. A ingestão insuficiente de macro e micronutrientes está estreitamente ligada ao estado nutricional, a limitada variedade de alimentos e a gravidade da sintomatologia associada ao transtorno ${ }^{8}$.

Embora, mesmo que esse público possua uma dieta variada e adequada sob a óptica nutricional, é necessário que o organismo execute algumas funções básicas, que infelizmente não se aplica nesse público, como: 1) digerir e quebrar adequadamente o alimento de forma absorvível; 2) absorver os nutrientes por meio do trato gastrointestinal saudável; 3) e converter os nutrientes a serem utilizados a nível celular ${ }^{9}$.

Pesquisa realizada no Brasil com 23 crianças com DEA de 3 a 10 anos verificou que o teor de carboidrato e proteínas esteve dentro da recomendação para a faixa etária, porém o de lipídeos não alcançou a recomendação desejada ${ }^{10}$.
Sendo válido destacar que dietas com essa insuficiência pode levar a redução da absorção de alguns micronutrientes, como as vitaminas lipossolúveis ${ }^{11}$.

\section{Má absorção intestinal e suas consequências}

Nos últimos anos, a Microbiota Gastrointestinal (MG) tem sido implicada como uma via potencial que afeta a manifestação dos sintomas em distúrbios cognitivos e do neurodesenvolvimento, assim como na resposta imunológica, possivelmente associada a mudanças metagenômicas que compromete a função da barreira intestinal $^{12}$

Além disso, pesquisadores sugerem que há associação de atraso no desenvolvimento comportamental por desordens gastrointestinais, como a diminuída produção de enzimas digestivas, inflamações da parede intestinal e a permeabilidade intestinal alterada, nessas crianças ${ }^{13}$.

Os problemas na $\mathrm{MG}$ revelaram maior gravidade no aspecto de irritabilidade, ansiedade, distúrbios do sono, transtornos de humor, intolerância alimentar e abstinência social, além de ter demonstrado esse grupo como menos propenso a responder $o$ tratamento com tendência a desenvolver comportamentos agressivos ${ }^{14,15}$.

Indivíduos com DEA que apresentam distúrbios gastrointestinais podem produzir mais citocinas pró-inflamatórias relacionadas à inflamação da mucosa e menos citocinas regulatórias, quando comparado a indivíduos com DEA que não apresentam essas alterações intestinais $^{12}$, assim como já ter sido relatado esses sintomas com a gravidade do $\mathrm{DEA}^{6}$.

Ressaltando que quando a digestão é indevida e o intestino muito permeável, os nutrientes não são adequadamente absorvidos, podendo afetar toda a função celular inclusive acarretar de forma prejudicial a função cerebral ${ }^{16}$. Além disso, o uso excessivo de antibióticos orais pode causar a perda desses nutrientes, uma vez que devido ao sistema imunológico debilitado podem alterar a flora intestinal ao resultar no crescimento excessivo da flora patogênica ${ }^{17}$. 


\section{Fibra alimentar e seu efeito na biodisponibilidade de micronutrientes}

Cada tipo de fibra alimentar (FA) exerce um efeito sobre a biodisponibilidade dos minerais, mas nem todos são afetados de forma igualitária. Enfatizando que a FA pode comprometer a biodisponibilidade do Cálcio, Zinco, Ferro e Magnésio, sobretudo quando essas fibras estão associadas a fitatos, oxalatos e substâncias fenólicas ${ }^{11}$.

Em estudo Brasileiro o consumo de FA foi avaliado em crianças com DEA, as quais apresentaram $38 \%$ em adequação, porém $27 \%$ de forma inadequada e $35 \%$ além da recomendação ${ }^{10}$. Já em estudo conduzido nos Estados Unidos verificou que crianças com DEA não atingiram a recomendação diária de FA, bem como de Cálcio e Ferro $^{18}$. Valendo ressaltar que esses estudos não especificaram a respeito do tipo de FA, o que ainda é muito limitado.

\section{Micronutrientes: status e sua relação com o DEA}

A suplementação vitamínica e/ou mineral vem sendo considerada o tratamento mais comumente usados para o DEA. Considerações específicas nesse público, como a característica e o comportamento do DEA, podem ser aumentadas ou, pelo menos, reduzir em maior risco devido ao estado nutricional sub-ótimo ${ }^{19}$. Além disso, crianças com DEA apresentaram mais insuficiências vitamínicas e minerais do que as crianças com desenvolvimento típico (DT) em pesquisa, e seus níveis estavam relacionados aos sintomas característicos do transtorno ${ }^{20}$.

Evidências relatam que crianças com DEA apresentam uma elevação do estresse oxidativo, principalmente devido à diminuição sérica dos níveis de glutationa, anormalidades metabólicas e nutricionais, incluindo problemas de sulfatação, metilação e disfunção mitocondrial ${ }^{19,21}$. Uma baixa concentração de enzimas antioxidantes séricas, nutrientes antioxidantes e níveis de glutationa assim como um aumento da concentração de próoxidantes que foram encontrados em estudos com esse público ${ }^{22}$. Além disso, pode resultar na redução de proteínas antioxidantes como transferrina e a ceruloplasmina, relacionando a perda da habilidade de linguagem ${ }^{23}$.
A disfunção mitocondrial no DEA pode resultar em deficiências cognitivas e comportamentais mais graves, maior propensão à regressão no desenvolvimento neurológico, convulsões e alterações gastrointestinais em comparação aos que não apresentam essa disfunção ${ }^{24}$.

As reações neuroimunes desempenham um papel patogênico em parte dos indivíduos com DEA, sendo provável que as anormalidades metabólicas de inflamação e estresse oxidativo estejam relacionadas às alterações de estrutura e função nestes pacientes, contribuindo para a diversidade de fenótipos, necessitando da compreensão do papel da neuroinflamação ${ }^{25,26}$. É relatado que o cérebro de um paciente com DEA, apresenta uma expressão alterada de genes associados à integridade da barreira hematoencefálica devido a um aumento desse processo inflamatório $^{27}$.

As crianças necessitam relativamente mais zinco em comparação aos adultos, tanto para o crescimento como para o desenvolvimento neurológico e cognitivo, ressaltando seu papel crucial no sistema imune, atuando como antiinflamatório, bem como no sistema de defesa antioxidante $^{11}$. Estudos têm demonstrado que um número considerável de crianças com DEA apresentaram pouca a grave deficiência desse mineral $^{28}$. Foi sugerido por pesquisadores uma possível alteração no funcionamento do sistema de expressão da metalotioneína neuroprotetora, processo crucial no metabolismo e proteção do acúmulo de metais pesados ${ }^{29}$, os quais estão relacionados a prejuízos cognitivos, comprometimento da atenção e maiores índices de distrações e impulsividade ${ }^{30}$.

Em indivíduos com DEA já foi evidenciado o diagnóstico de hipovitaminose A relacionada ao consumo alimentar inadequado ${ }^{10}$. O que se faz importante uma adequada ingestão de zinco uma vez que, uma inadequada ingestão de vitamina A afeta a função de zinco mesmo que este mineral seja consumido em quantidades suficientes, sendo válido pontuar que essa deficiência pode resultar em falha no crescimento ${ }^{11}$.

Um estudo realizado na China examinou por meio de avaliações dietéticas e questionários o comportamento alimentar de 154 crianças com 
DEA e 73 crianças com DT, verificando que o grupo com DEA apresentou baixo nível sérico de vitamina $A$, podendo ser um fator de risco para o agravamento dos sintomas típicos do distúrbio ${ }^{31}$. Pesquisadores sugerem que essa vitamina em alta concentração pode ser um fator de proteção para crianças com autismo, visto que sua deficiência estava negativamente relacionada a escala de classificação comportamental e de responsabilidade social. A respeito das subescalas, retraimento social, fala inapropriada, vida cotidiana retardatária e comportamento estereotipado mostraram escores significativamente maiores no grupo com deficiência grave de vitamina A em comparação aos com nível normal dessa vitamina ${ }^{20}$.

A adequação da vitamina A é indispensável para o comportamento no DEA devido a regulação da: 1) função neurossináptica via o metabólito bioativo do ácido retinóico; 2) plasticidade sináptica homeostática do eixo da diferenciação 38-oxitocina; 3) e homeostase da energia mitocondrial ${ }^{32,33}$.

Em estudo com 53 crianças com DEA e 53 crianças com DT, na faixa etária de 4 a 6 anos de idade, verificou que os níveis séricos de cálcio, vitamina $A$ e folato estavam mais baixos nos indivíduos com DEA do que nos controles ${ }^{34}$. Corroborando com os resultados encontrados em estudo realizado na Espanha que apontaram 5 vezes mais risco de crianças com DEA, comparado com as crianças controle de não atingirem a meta recomendada de cálcio e ferro e 3 vezes mais risco no alcance de vitamina $C^{35}$.

Pesquisadores compararam o consumo alimentar por meio do inquérito Recordatório 24 horas $(\mathrm{R} 24 \mathrm{H})$ e grupos alimentares por faixa etária, encontrando um maior consumo de vitamina B6 e E nos indivíduos com DEA em comparação aos de DT, e um menor consumo de cálcio no grupo de crianças com $\mathrm{DEA}^{18}$. A vitamina $\mathrm{B} 6$ em altas dosagens pode apresentar danos neurais como a perda da mielina e degeneração de fibras sensoriais dos nervos periféricos e a vitamina $\mathrm{E}$, por sua vez, tem função neuroprotetora, apresentando efeitos inofensivos diante de altas dosagens ${ }^{11}$.

Outro estudo usando o mesmo método de avaliação identificou que crianças com DEA, com faixa etária de 6 a 9 anos, não alcançaram as recomendações dietéticas para vitamina $\mathrm{C}$ e $\mathrm{E}$ em comparação com os de $\mathrm{DT}^{36}$. Ressaltando que a vitamina $\mathrm{C}$ em deficiência pode estar associada com apatia, mudança na personalidade e no desenvolvimento psicomotor, atribuídos ao prejuízo na síntese de catecolaminas, como resultado da baixa atividade da dopamina $\beta$-hidroxilase ${ }^{11}$, sintomas esses característicos no DEA.

Foi avaliado o consumo alimentar nesse público e averiguou um menor consumo de cálcio, fósforo, selênio, tiamina, riboflavina e vitamina $\mathrm{B} 12$ e um maior consumo de vitamina $\mathrm{E}^{37}$. Portanto, já foi evidenciado que o fósforo em excesso se liga a minerais como o cálcio, magnésio, potássio, zinco, cromo e vitaminas hidrossolúveis, tornandoos indisponíveis ${ }^{16}$.

Em estudo com crianças com DEA foi verificada possível redução de $50 \%$ e $69,2 \%$ no consumo de cálcio e sódio, respectivamente, e o magnésio apresentando-se com consumo nocivo além do recomendado em $58 \%$ das crianças avaliadas ${ }^{10}$. Em virtude do maior envolvimento do magnésio nas funções neurológicas, seus níveis elevados no plasma podem causar efeitos adversos, tornando-se muito grave ${ }^{11}$.

A redução do consumo de cálcio está diretamente ligada a diversas funções orgânicas como modulação de sinais de transdução e metabolismo de produção de energia e proliferação celular. Alguns sintomas resultantes dessa deficiência são: 1) ansiedade; 2) hiperatividade; 3) agitação; 4) alucinações; 5) irritabilidade; 6) nervosismo; 7) agressão; 8) estresse crônico; 9) e dificuldade de aprendizagem ${ }^{11}$, sendo evidenciado em crianças com DEA podendo refletir no seu comportamento $^{10,18,34,35}$. Enfatizando seu envolvimento da atividade de transportar o aspartato/glutamato e no estresse oxidativo em cérebros autistas ${ }^{38}$.

As concentrações de Cálcio, Magnésio, Ferro, Zinco e Folato foram positivamente correlacionadas com comportamento adaptativo, motor grosso, motor fino, linguagem $\mathrm{e}$ comportamento pessoal-social no $\mathrm{DEA}^{20}$. Disfunção mitocondrial, inflamação, desregulação imune, e oxidativa indicam a base biológica para os problemas comportamentais relatados em indivíduos com DEA, o que visa avaliar o tratamento de intervenção vitamínica e de minerais $^{24,39}$. A deficiência de ferro está estreitamente ligada a alteração cognitiva com 
déficit de atenção e problemas relacionados ao sono $^{20}$.

O folato e o vitamina B12 são indispensáveis para o desenvolvimento neuronal normal, função e deficiências graves destas vitaminas e podem afetar diretamente a função cerebral $^{11}$. Foi encontrado menores valores dessas vitaminas em dieta e soro em crianças com DEA em comparação com crianças de DT, sendo o folato associado ao comportamento adaptativo, motor grosso, linguagem e comportamento social em estudo $^{20,40}$. Sua suplementação foi sugerida ser mais efetiva no DEA severo de idade inferior a 3 anos, que apresentarem baixos níveis de folato cerebral, o que resultaria melhorias comportamentais, motoras e neurológicas ${ }^{41}$.

A respeito da vitamina $\mathrm{D}$, pesquisadores relatam que as inflamações intestinais podem influenciar seus níveis plasmáticos ${ }^{42}$. E há considerações sobre seu envolvimento na proliferação e diferenciação celular, neurotropismo, neuroproteção, neurotransmissão, mielinização e neuroplasticidade ${ }^{43}$. Em estudo conduzido no Brasil os níveis séricos de 25-hidroxivitamina $\mathrm{D}$ foram menores em crianças com DEA $(26,48 \pm 3,48 \mathrm{ng}$ mL-1) quando comparados aos indivíduos em DT $\left(40,52 \pm 3,13\right.$ ng mL-1) $(p<0,001)^{44}$. Corroborando com resultados de pesquisas na China $^{45} \mathrm{e}$ Dinamarca $^{46}$.

Nutrientes como a vitamina B6 também são de extrema importância para a metilação, transulfatação e sulfatação, que representam um conjunto de atividades bioquímicas que não funcionam adequadamente nos portadores com DEA. Quando há limitação dessas transformações metabólicas, os neurotransmissores não são adequadamente ativados, ocasionando sintomas de ansiedade, depressão, déficit de atenção e transtorno do sono ${ }^{20}$. Que quando associado ao maior consumo de alumínio, mercúrio, glutamato e outras substâncias artificiais ingeridas por meio da alimentação, pode contribuir para seu acúmulo resultando em alterações cerebrais como irritabilidade, agressividade e hiperatividade ${ }^{10}$.

Estudo avaliou o estado nutricional e metabólico no DEA em comparação com grupo de DT, ambos com faixa etária de 5 a 16 anos, destacando que a maioria apresentou status de micronutrientes dentro do intervalo de referência, mas em contrapartida, foi identificado um aumento do estresse oxidativo, capacidade reduzida de transporte de energia, sulfatação e desintoxicação. Sugerindo que o suporte nutricional com a suplementação aumentada da ingestão de nutrientes pode reduzir os sintomas e comorbidades associadas ao distúrbio ${ }^{47}$. $\mathrm{O}$ que foi evidenciado em outro estudo a melhora da maioria dos biomarcadores metabólicos com suplementação de vitaminas e minerais por um período de 3 meses em comparação com o grupo controle ${ }^{48}$.

Com o intuito de comparar os resultados do gerenciamento de intervenção medicamentosa com a suplementação de micronutrientes nos sintomas do DEA, pesquisadores concluíram que ambos os grupos melhoraram, mas a melhora do grupo de micronutrientes foi significativamente maior, resultando em vantagens como: 1) menor nível de atividade; 2) menos retração social; 3) menos sentimento de raiva e irritabilidade; 4) melhor espontaneidade; 5) menos eventos adversos; 6) e menos ganho de peso ${ }^{49}$.

Apesar da escassez de dados que fazem menção a essa relação dos micronutrientes com a sintomatologia comportamental no DEA, observase que esse público tem uma maior probabilidade de ter uma inadequação de micronutrientes, em que pode ser heterogêneo, variando de deficiência a toxicidade, ocasionada pela dieta restritiva e monótona, refletindo assim no seu estado nutricional.

Pontuando ainda que a frequência de sintomas intestinais pode estar atrelada ao agravamento do comportamento alimentar e da sintomatologia típica do transtorno, que consequentemente reflete de forma negativa na absorção desses nutrientes. Sendo válido destacar que alguns desses comportamentos podem ser oriundos das alterações dos níveis de vitaminas e minerais, uma vez que desempenham papéis cruciais no neurodesenvolvimento dessas crianças.

\section{Conclusão}

É necessário que sejam feitas novas pesquisas nesta área para avaliar o consumo dietético de micronutrientes em específico, verificar se há comprometimento da absorção desses nutrientes, seu impacto no comportamento 
característico e na eficácia da suplementação validadas nesse público. Assim, possibilitando a criação de medidas preventivas, embora as causas do DEA ainda sejam desconhecidas, a influência direta dos micronutrientes não deve ser negligenciada, portanto essencial, principalmente no período crítico do desenvolvimento neural no terceiro trimestre da gravidez, bem como suas relações com a microbiota intestinal.

\section{Referências}

1. Lombardi VC, Meirleir KLD, Subramanian K, Nourani SM, Dagda RK, Delaney SL, et al. Nutritional Modulation of the Intestinal Microbiota; Future Opportunities for the Prevention and Treatment of Neuroimmune and Neuroinflammatory Disease. The Journal of nutritional biochemistry, 2018. disorders (ASDs). Microbiome, 2018, 6.1: 133.

2. Siqueira CC, Ferreira EO, Bittencourt RG, Cavalheiro FR, de Abreu Silveira JA, Santos MFR. O cérebro autista: a biologia da mente e sua implicação no comprometimento social. Revista Transformar, 2016, 8(8), 221-237.

3. Meguid N, Anwar M, Zaki S, Kandeel W, Ahmed N, Tewfik I. Dietary patterns of children with autism spectrum disorder: a study based in Egypt. Open access Macedonian journal of medical sciences, 2015, 3(2), 262.

4. Ames, B. N., Elson-Schwab, I., \& Silver, E. A. (2002). High-dose vitamin therapy stimulates variant enzymes with decreased coenzyme binding affinity (increased $\mathrm{K}$ $\mathrm{m})$ : relevance to genetic disease and polymorphisms. The American journal of clinical nutrition, 75(4), 616658.

5. Rapin, I., \& Goldman, S. (2008). A escala CARS brasileira: uma ferramenta de triagem padronizada para o autismo. Jornal de Pediatria, 84(6), 473-475.

6. Buie T, Campbell DB, Fuchs GJ, Furuta GT, Levy J, Vandewater J, et al. Evaluation, diagnosis, and treatment of gastrointestinal disorders in individuals with ASDs: a consensus report. Pediatrics 125 (Suppl 1), 2010, S1S18.

7. Silva NID. Relação entre hábito alimentar e síndrome do espectro autista. Doctoral dissertation, Universidade de São Paulo, 2011.

8. Zuchetto AT, Cavalcante TM, Pimenta RA, Zanon PA, Nasser JP. Avaliação da composição corporal de crianças e jovens com deficiência. Revista Portuguesa de Ciências do Desporto, 2014.
9. Reichow B. Overview of meta-analyses on early intensive behavioral intervention for young children with autism spectrum disorders. Journal of autism and developmental disorders, 2012, 42(4), 512-520.

10. Caetano MV, Gurgel DC. Perfil nutricional de crianças portadoras do transtorno do espectro autista. Revista Brasileira em Promoção da Saúde, 2018, 31(1).

11. Cozzolino SMF. Biodisponibilidade de nutrientes. São Paulo: Manole; 2005.

12. Rose DR, Yang H, Serena G, Sturgeon C, Ma B, Careaga $\mathrm{M}$, et al. Differential immune responses and microbiota profiles in children with autism spectrum disorders and co-morbid gastrointestinal symptoms. Brain, behavior, and immunity, 2018, 70, 354-368.

13. Frye RE, Rose S, Slattery J, MacFabe DF. Gastrointestinal dysfunction in autism spectrum disorder: the role of the mitochondria and the enteric microbiome. Microbial ecology in health and disease, 2015, 26(1), 27458.

14. Nikolov RN, Bearss KE, Lettinga J, Erickson C, Rodowski M, Aman MG, et al. Gastrointestinal symptoms in a sample of children with pervasive developmental disorders. Journal of autism and developmental disorders, 2009, 39(3), 405-413.

15. Mazurek MO, Vasa RA, Kalb LG, Kanne SM, Rosenberg D, Keefer A, et al. Anxiety, sensory overresponsivity, and gastrointestinal problems in children with autism spectrum disorders. Journal of Abnormal Child Psychology, 2013, 41, 165-176.

16. Marcelino C. Autismo: a esperança pela nutrição. Rio e Janeiro, Mbooks; 2010.

17. Adams JB, Johansen LJ, Powell LD, Quig D, Rubin RA. Gastrointestinal flora and gastrointestinal status in children with autism-comparisons to typical children and correlation with autism severity. BMC gastroenterology, 2011, 11.1: 22.

18. Herndon AC, DiGuiseppi C, Johnson SL, Leiferman J, Reynolds A. Does nutritional intake differ between children with autism spectrum disorders and children with typical development?. Journal of autism and developmental disorders, 2009, 39(2), 212.

19. Bjørklund G, Waly MI, Al-Farsi Y, Saad K, Dadar M, Rahman MM, et al. The Role of Vitamins in Autism Spectrum Disorder: What Do We Know? Journal of Molecular Neuroscience, 2019, 1-15.

20. Guo M, Li L, Zhang Q, Chen L, Dai Y, Liu L, et al. Vitamin and mineral status of children with autism spectrum disorder in Hainan Province of China: associations with symptoms. Nutritional neuroscience, 2018, 1-8. 
21. James SJ, Cutler P, Melnyk S, Jerniga S, Janak L, Gaylor DW, Neubrander JA. Metabolic biomarkers of increased oxidative stress and impaired methylation capacity in children with autism. The American journal of clinical nutrition, 2004, 80(6), 1611-1617.

22. McGinnis WR. Oxidative stress in autism. Alternative Therapies in Health and Medicine, 2004,10(6), 22-36.

23. Chauhan A, Chauhan V (2006). Oxidative stress in autism. Pathophysiology, 13(3), 171-181.

24. Rossignol DA, Frye RE. Evidence linking oxidative stress, mitochondrial dysfunction, and inflammation in the brain of individuals with autism. Frontiers in physiology, 2014, 5, 150.

25. Herbert MR. Large brains in autism: the challenge of pervasive abnormality. The Neuroscientist, 2005, 11(5), 417-440.

26. Pardo CA, Vargas DL, Zimmerman AW. Immunity, neuroglia and neuroinflammation in autism. International review of psychiatry, 2005, 17(6), 485-495.

27. Fiorentino M, Sapone A, Senger S, Camhi SS, Kadzielski SM, Buie TM, Fasano A. Blood-brain barrier and intestinal epithelial barrier alterations in autism spectrum disorders. Molecular autism, 2016, 7(1), 49.

28. Yasuda H, Yoshida K, Yasuda Y, Tsutsui T. Infantile zinc deficiency: association with autism spectrum disorders. Scientific reports, 2011, 1, 129.

29. Crăciun EC, Bjørklund G, Tinkov AA, Urbina MA, Skalny AV, Rad F, Dronca E. Evaluation of whole blood zinc and copper levels in children with autism spectrum disorder. Metabolic brain disease, 2016, 31(4), 887-890.

30. Dumcke TS (2018). Associação entre exposição a resíduos e dificuldades emocionais e comportamentais de escolares.

31. Liu X, Liu J, Xiong X, Yang T, Hou N, Liang X, et al. Correlation between nutrition and symptoms: nutritional survey of children with autism spectrum disorder in Chongqing, China. Nutrients, 2016, 8(5), 294.

32. Lai X, Wu X, Hou N, Liu S, Li Q, Yang T, et al. Vitamin A deficiency induces autistic-like behaviors in rats by regulating the RARbeta-CD38-oxytocin axis in the hypothalamus. Mol Nutr Food Res 2018;62(5). doi:10.1002/mnfr.201700754.

33. Xu X, Li C, Gao X, Xia K, Guo H, Li Y, et al. Excessive UBE3A dosage impairs retinoic acid signaling and synaptic plasticity in autism spectrum disorders. Cell Res. 2018;28:48-68.

34. Sun C, Xia W, Zhao Y, Li N, Zhao D, Wu L. Nutritional status survey of children with autism and typically developing children aged 4-6 years in Heilongjiang Province, China. Journal of nutritional science, 2013, 2.

35. Marí-Bauset S, Llopis-González A, Zazpe-García I, Marí-Sanchis A, Morales-Suárez-Varela M. Nutritional status of children with autism spectrum disorders (ASDs): a case-control study. Journal of autism and developmental disorders, 2015, 45(1), 203-212.

36. Marí-Bauset S, Llopis-González A, Zazpe I, MaríSanchis A, Morales Suárez-Varela M. Comparison of nutritional status between children with autism spectrum disorder and typically developing children in the Mediterranean Region (Valencia, Spain). Autism, 2017, 21(3), 310-322.

37. Esteban-Figuerola $\mathrm{P}$, Canals J, Fernández-Cao JC, Arija Val V. Differences in food consumption and nutritional intake between children with autism spectrum disorders and typically developing children: A metaanalysis. Autism, 2018, 1362361318794179.

38. Palmieri L, Papaleo V, Porcelli V, Scarcia P, Gaita L, Sacco R, Militerni R. Altered calcium homeostasis in autism-spectrum disorders: evidence from biochemical and genetic studies of the mitochondrial aspartate/glutamate carrier AGC1. Molecular psychiatry, 2010, 15(1), 38.

39. Goh S, Dong Z, Zhang Y, DiMauro S, Peterson BS. Mitochondrial dysfunction as a neurobiological subtype of autism spectrum disorder: evidence from brain imaging. JAMA psychiatry, 2014, 71(6), 665-671.

40. Al-Farsi YM, Waly MI, Deth RC, Al-Sharbati MM, Al-Shafaee M, Al-Farsi O, Al-Adawi S. Low folate and vitamin B12 nourishment is common in Omani children with newly diagnosed autism. Nutrition, 2013, 29(3), 537-541.

41. Main PA, Angley MT, Thomas P, O'Doherty CE, Fenech M. Folate and methionine metabolism in autism: a systematic review. Am J Clin Nutr. 2010; 91(6):1598620.

42. Bours PH, Wielders JP, Vermeijden JR, van de Wiel A. Seasonal variation of serum 25-hydroxyvitamin D levels in adult patients with inflammatory bowel disease. Osteoporosis international : a journal established as result of cooperation between the European Foundation for Osteoporosis and the National Osteoporosis Foundation of the USA. 2011; 22(11):2857-67.

43. De Luca, GC Kimball SM, Kolasinski J, Ramagopalan SV, Ebers GC Review: the role of vitamin $\mathrm{D}$ in nervous system health and disease. Neuropathol Appl Neurobiol 2013 Aug;(39):458-484

44. Tostes MH, Polonini HC, Gattaz WF, Raposo NR, Baptista EB. Low serum levels of 25-hydroxyvitamin D 
(25-OHD) in children with autism. Trends in Psychiatric and Psychoterapy 2012 Sep (34) 3:161-3.

45. Gong ZL, Luo CM, Wang L, Shen L, Wei F, Tong RJ, Liu Y. Serum 25-hydroxyvitamin D levels in Chinese children with autism spectrum disorders.NeuroReport 2014 Jan 8.25(1):23-7.

46. KocovskA E, Andorsdóttir G, Weihe P, at al. Vitamin $\mathrm{d}$ in the general population of young adults with autism in the faroe islands.J.Autism Dev. Disord 2014 Dec 44(12): 2996-3005.

47. Adams JB, Audhya T, McDonough-Means S, Rubin RA, Quig D, Geis E, et al. Nutritional and metabolic status of children with autism vs. neurotypical children, and the association with autism severity. Nutrition \& metabolism. 2011; 8(1):34.

48. Adams JB, Audhya T, McDonough-Means S, Rubin
RA, Quig D, Geis E, et al. Effect of a vitamin/mineral supplement on children and adults with autism. BMC pediatrics. 2011; 11:111.

49. Mehl-Madrona L, Leung B, Kennedy C, Paul S, Kaplan BJ. Micronutrients versus standard medication management in autism: a naturalistic case-control study. Journal of child and adolescent psychopharmacology. 2010; 20(2):95-103.

Autor para correspondência: Nathália de Freitas Penaforte nutripenaforte@gmail.com 